\title{
Bi-Space Shift Keying Modulation for MIMO Systems
}

\author{
Han-Wen Liang, Ronald Y. Chang, Wei-Ho Chung, Hongke Zhang, and Sy-Yen Kuo
}

\begin{abstract}
In this letter, we propose a new Space Shift Keying (SSK)-based modulation that maps the information onto the antenna indices. The proposed Bi-Space Shift Keying (BiSSK) scheme extends SSK by employing a dual set of antenna indices, one associated with a real number and the other with an imaginary number. The proposed BiSSK preserves the advantages of low receiver complexity of SSK and yet doubles its transmission rates, with negligible performance penalty. The proposed BiSSK outperforms Generalized Space Shift Keying (GSSK) at the same given target transmission rate, as demonstrated by both errorprobability analyses and simulations.
\end{abstract}

Index Terms-Spatial modulation (SM), space shift keying (SSK), multiple input multiple output (MIMO), maximum likelihood (ML) detection, performance analysis.

\section{INTRODUCTION}

$\mathbf{U}$ SING antenna indices as a dimension for modulation in Multiple-Input Multiple-output (MIMO) wireless communication system has received increasing research interests in recent years. With varying the indices of activated and idle antennas to form a set of transmission patterns, the information is fully or partially mapped onto the patterns when spatial dimension is used for modulation. The Space Shift Keying (SSK), mapping the information onto only the antenna indices, is advantageous compared to conventional MIMO in the aspects of reduced hardware expenses (fewer required Radio Frequency (RF) chains), the low detection complexity, and less stringent transceiver requirements due to the absence of Amplitude/Phase Modulation (APM) [1]. The spatial dimension can also be jointly designed with other existing techniques to improve performance, such as SpaceTime Block Coding (STBC) [2], [3] and power allocation [4]. In APM constellation, the modulation space is equivalent to the one-dimensional complex space formed by a column of each channel realization [1] (see Sec. II). In contrast to APM, the modulation space in SSK is the $N_{r}$-dimensional space, where $N_{r}$ is the number of receiving antennas. Therefore,

Manuscript received February 29, 2012. The associate editor coordinating the review of this letter and approving it for publication was S. Jin.

This research was supported by the National Science Council, Taiwan under Grant NSC 99-2221-E-002-108-MY3, the National Natural Science Foundation of China (NSFC) under Grant 60833002, and the 111 Project under Grant B08002.

H.-W. Liang is with the Department of Electrical Engineering, National Taiwan University, and the Research Center for Info. Tech. Innovation, Academia Sinica.

R. Y. Chang and W.-H. Chung (corresponding author) are with the Research Center for Info. Tech. Innovation, Academia Sinica (e-mail: whc@citi.sinica.edu.tw).

H. Zhang is with the College of Electronics and Info. Engineering, Beijing Jiaotong University.

S.-Y. Kuo is with the Department of Electrical Engineering, National Taiwan University, and the College of Electronics and Info. Engineering, Beijing Jiaotong University.

Digital Object Identifier 10.1109/LCOMM.2012.061912.120448 the performance of SSK may outperform APM since the SSK constellation points are farther from each other and less sensitive to noise.

A critical issue associated with the design for spatial modulation relates to the construction of transmission patterns. Due to the size of a mobile device, the number of transmitting antennas is not always a power of two. Furthermore, due to the limited number of transmitting antennas, the transmission rate of SSK is limited. Fractional Bit Encoded Spatial Modulation (FBE-SM) [5] was proposed to encode a block of binary-represented information to a block of arbitrary numberrepresented digits. Spatial Modulation (SM) [6], mapping the information to both the spatial dimension and the APM dimension, is one of the potential solutions to mitigate the antenna limitations (equivalent to improving transmission rate) of SSK. However, performing modulation in the APM dimension has more stringent transceiver requirements. The SSK-based modulation schemes employ only the spatial dimension to encode the information. Generalized Space Shift Keying (GSSK) [7], an SSK-based modulation scheme using combinations of the antenna indices to form a set of transmission patterns, is another viable solution to mitigate the antenna limitations of SSK. Hamming code-aided Space Shift Keying (HSSK) [8] proposed a sophisticated constellation design to offer a rich design options, which achieves better transmission rate, performance, and power tradeoffs.

This letter proposes a new SSK-based modulation scheme, which uses a dual set of antenna indices, associated with real and imaginary numbers, respectively, to carry information simultaneously. The proposed scheme, termed Bi-Space Shift Keying (BiSSK), has the same benefits of SSK such as low detection complexity and few RF chains while doubling the transmission rate of SSK. Compared to GSSK, the proposed BiSSK requires fewer RF chains and achieves better performance due to a better utilization of the modulation space. The error probability and the diversity of BiSSK are analytically derived, and the simulation results demonstrate the accuracy of the analysis and the performance advantages of the proposed BiSSK scheme.

This letter is organized as follows. Sec. II gives the system model. Sec. III describes the proposed BiSSK scheme. The analytical error probability and diversity are derived in Sec. IV. The simulations are given in Sec. V, and Sec. VI concludes the proposed work.

\section{System MODEL}

We consider an uncoded MIMO transmission system with $N_{t}$ transmitting antennas and $N_{r}$ receiving antennas employing SSK-based modulation. The baseband signal model is given by

$$
\boldsymbol{y}=\sqrt{\rho} \boldsymbol{H} \boldsymbol{x}+\boldsymbol{n} .
$$


TABLE I

EXAMPLE OF BISSK SYMBOL MAPPING FOR 2-BIT TRANSMISSION IN A SYSTEM WITH $N_{t}=2$

\begin{tabular}{cc}
\hline $\boldsymbol{s}=\left[\begin{array}{ll}\boldsymbol{s}_{\mathbf{1}} & \boldsymbol{s}_{2}\end{array}\right]$ & \multicolumn{3}{c}{$\boldsymbol{w}$} \\
\hline$\left[\begin{array}{ll}0 & 0\end{array}\right]$ & {$\left[\begin{array}{lll}1+j & 0\end{array}\right]^{T}$} \\
{$\left[\begin{array}{ll}0 & 1\end{array}\right]$} & {$\left[\begin{array}{rrr}1 & j\end{array}\right]^{T}$} \\
{$\left[\begin{array}{ll}1 & 0\end{array}\right]$} & {$\left[\begin{array}{rrr}j & 1\end{array}\right]^{T}$} \\
{$\left[\begin{array}{ll}1 & 1\end{array}\right]$} & {$\left[\begin{array}{rr}0 & 1+j\end{array}\right]^{T}$} \\
\hline
\end{tabular}

The transmitted symbol $\boldsymbol{x}$ is an $N_{t} \times 1$ vector that has $n_{t}$ nonzeros and $N_{t}-n_{t}$ zeros, where the selection of $n_{t}$ depends on the specific SSK-based modulation used. The received symbol is $\boldsymbol{y} \in \mathbb{C}^{N_{r} \times 1}$. The flat-fading channel $\boldsymbol{H} \in \mathbb{C}^{N_{r} \times N_{t}}$ has independent and identically distributed (i.i.d.) zero-mean complex Gaussian random variable entries and covariance matrix $\sigma_{H}^{2} \boldsymbol{I}_{N_{r}}$, where $\sigma_{H}^{2}=1$ and $\boldsymbol{I}_{M}$ represents the $M \times M$ identity matrix. The Additive White Gaussian Noise (AWGN) $\boldsymbol{n}$ has zero-mean and covariance matrix $N_{0} \boldsymbol{I}_{N_{r}}$. The power amplification factor $\rho$ is defined as $E_{s} / \mathbb{E}\left[\boldsymbol{x}^{H} \boldsymbol{x}\right]$, where $\mathbb{E}[\cdot]$ denotes the expectation operation and $E_{s}$ represents the symbol energy.

For SSK modulation, only one transmitting antenna is activated at a time. Therefore, the attainable symbol rate is $\left\lfloor\log _{2} N_{t}\right\rfloor$ bits for SSK, where $\lfloor\cdot\rfloor$ is the floor operation giving the largest integer not greater than its argument. For GSSK, since $n_{t}$ transmitting antennas are activated simultaneously, the attainable symbol rate is $\left\lfloor\log _{2}\left(\begin{array}{l}N_{t} \\ n_{t}\end{array}\right)\right\rfloor$ bits.

\section{THE PROPOSED BISSK}

The main idea of BiSSK is employing a dual set of antenna indices, one associated with a real number and the other with an imaginary number, to convey information. Given $N_{t}$ transmitting antennas, the set of available SSK symbol candidates is $\mathcal{A}_{S S K}^{*} \triangleq\left\{\boldsymbol{x}=\left[x_{1}, x_{2}, \cdots, x_{N_{t}}\right]^{T} \mid x_{i} \in\{0,1\}, \sum_{i} x_{i}=1\right\}$, where the superscript $T$ denotes the transpose operation. The SSK alphabet $\mathcal{A}_{S S K}$, mapped by $\left\lfloor\log _{2} N_{t}\right\rfloor$ bits of information, is a subset of $\mathcal{A}_{S S K}^{*}$. The BiSSK alphabet is defined as $\mathcal{A} \triangleq\left\{\boldsymbol{w}=\boldsymbol{x}_{\boldsymbol{R}}+j \boldsymbol{x}_{\boldsymbol{I}} \mid \boldsymbol{x}_{\boldsymbol{R}}, \boldsymbol{x}_{\boldsymbol{I}} \in \mathcal{A}_{S S K}\right\}$. As can be seen, BiSSK can transmit twice as much data as SSK where only one index set is used. The BiSSK symbol mapping rule is elaborated as follows.

1) Divide the information bits $s$ into two equal-sized parts $s_{1}$ and $s_{2}$.

2) Map $s_{1}$ and $s_{2}$ to two $N_{t} \times 1$ symbols $\boldsymbol{x}_{\boldsymbol{R}}$ and $\boldsymbol{x}_{\boldsymbol{I}}$ by standard SSK mapping, respectively.

3) Sum up $\boldsymbol{x}_{\boldsymbol{R}}$ and $j \boldsymbol{x}_{\boldsymbol{I}}$ entrywise to form the BiSSK symbol $\boldsymbol{w}$.

For example, in a system with $N_{t}=2$ using BiSSK to transmit 2-bit information $s=\left[\begin{array}{ll}0 & 1\end{array}\right]$, we first map $s_{1}=[0]$ to symbol $\left[\begin{array}{ll}1 & 0\end{array}\right]^{T}$ and $\boldsymbol{s}_{\mathbf{2}}=$ [1] to symbol $\left[\begin{array}{ll}0 & j\end{array}\right]^{T}$, and then sum up the two mapped symbols to form the BiSSK symbol $[1 j]^{T}$. The full symbol mapping for this example is summarized in Table I for the proposed BiSSK.

If BiSSK is employed, the power amplification factor in (1) can be specifically denoted as $\rho_{B}=E_{s} / 2$. The optimal detection of BiSSK modulated symbol $\overline{\boldsymbol{w}}$ at the receiver is hence given by the maximum likelyhood (ML) criterion, i.e.,

$$
\overline{\boldsymbol{w}}=\arg \min _{\boldsymbol{w} \in \mathcal{A}}\left\|\boldsymbol{y}-\sqrt{\rho_{B}} \boldsymbol{H} \boldsymbol{w}\right\|^{2},
$$

where $\|\cdot\|$ represents the Euclidean norm.

\section{ERror Probability AND DiVERSITY DERIVATION}

Error probability: Consider an uncoded system employing ML detection at the receiver. By applying the union bound technique to the pairwise error probability (PEP) of deciding on $\boldsymbol{w}_{j}$ given that $\boldsymbol{w}_{\boldsymbol{i}}$ is transmitted, the symbol error rate (SER) for BiSSK modulation can be approximated as

$$
\begin{aligned}
P_{\text {sym }} & =\mathbb{E}_{\boldsymbol{w}_{\boldsymbol{i}}}\left[\bigcup_{\boldsymbol{w}_{\boldsymbol{j}}} P\left(\boldsymbol{w}_{\boldsymbol{i}} \rightarrow \boldsymbol{w}_{\boldsymbol{j}}\right)\right] \\
& \leq \frac{1}{N_{t}^{2}} \sum_{\boldsymbol{w}_{\boldsymbol{i}}} \sum_{\boldsymbol{w}_{\boldsymbol{j}} \neq \boldsymbol{w}_{\boldsymbol{i}}} P\left(\boldsymbol{w}_{\boldsymbol{i}} \rightarrow \boldsymbol{w}_{\boldsymbol{j}}\right) \\
& =\sum_{\boldsymbol{w}_{\boldsymbol{j}} \neq \boldsymbol{w}_{\boldsymbol{i}}} P\left(\boldsymbol{w}_{\boldsymbol{i}} \rightarrow \boldsymbol{w}_{\boldsymbol{j}}\right),
\end{aligned}
$$

where possible transmitted symbols $\boldsymbol{w}_{\boldsymbol{i}} \in \mathcal{A}$ are averaged to derive the SER. We use $m$ and $n$ ( $p$ and $q$ ) to represent the antenna indices of $\boldsymbol{w}_{\boldsymbol{i}}\left(\boldsymbol{w}_{\boldsymbol{j}}\right)$ associated with the real and imaginary numbers, respectively, i.e.,

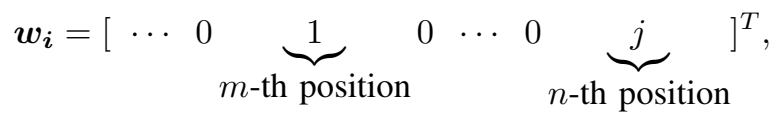

and

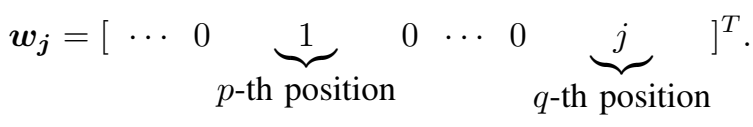

Then, we separate the set of all possible error symbols $\mathcal{A} \backslash \boldsymbol{w}_{\boldsymbol{i}}$ into two non-overlapping subsets $\mathcal{W}^{\prime}$ and $\mathcal{W}^{\prime \prime}$ according to the relation of $\boldsymbol{w}_{\boldsymbol{i}}$ and the candidate error symbol $\boldsymbol{w}_{\boldsymbol{j}}$ in terms of $(m, n, p, q)$. Specifically, $\mathcal{W}^{\prime}=\left\{\boldsymbol{w}_{\boldsymbol{j}} \in \mathcal{A} \backslash \boldsymbol{w}_{\boldsymbol{i}} \mid(p=m, q \neq\right.$ $n)$ or $(p \neq m, q=n)\}$ and $\mathcal{W}^{\prime \prime}=\left\{\boldsymbol{w}_{\boldsymbol{j}} \in \mathcal{A} \backslash \boldsymbol{w}_{\boldsymbol{i}} \mid(p \neq\right.$ $m, q \neq n)\}$. The cardinalities of the two subsets are $\left|\mathcal{W}^{\prime}\right|=$ $2\left(N_{t}-1\right)$ and $\left|\mathcal{W}^{\prime \prime}\right|=\left(N_{t}-1\right)^{2}$. As a result, (3) can be rewritten as

$$
\begin{aligned}
P_{\text {Sym }} & \leq \sum_{\boldsymbol{w}_{\boldsymbol{j}} \neq \boldsymbol{w}_{\boldsymbol{i}}} P\left(\boldsymbol{w}_{\boldsymbol{i}} \rightarrow \boldsymbol{w}_{\boldsymbol{j}}\right) \\
& =\sum_{\boldsymbol{w}_{\boldsymbol{j}} \in \mathcal{W}^{\prime}} P\left(\boldsymbol{w}_{\boldsymbol{i}} \rightarrow \boldsymbol{w}_{\boldsymbol{j}}\right)+\sum_{\boldsymbol{w}_{\boldsymbol{j}} \in \mathcal{W}^{\prime \prime}} P\left(\boldsymbol{w}_{\boldsymbol{i}} \rightarrow \boldsymbol{w}_{\boldsymbol{j}}\right) .
\end{aligned}
$$

The PEP in the two summation terms in (4) is derived as follows. First, the PEP conditioned on $\boldsymbol{H}$ is given by [1]

$$
\begin{aligned}
P\left(\boldsymbol{w}_{\boldsymbol{i}} \rightarrow \boldsymbol{w}_{\boldsymbol{j}} \mid \boldsymbol{H}\right) & =P\left(\begin{array}{c}
\left\|\boldsymbol{y}-\sqrt{\rho_{B}} \boldsymbol{H} \boldsymbol{w}_{\boldsymbol{i}}\right\|^{2} \\
>\left\|\boldsymbol{y}-\sqrt{\rho_{B}} \boldsymbol{H} \boldsymbol{w}_{\boldsymbol{j}}\right\|^{2}
\end{array}\right) \\
& =P\left(\begin{array}{c}
\Re\left\{\boldsymbol{n}^{\boldsymbol{H}}\left(\boldsymbol{H} \boldsymbol{w}_{\boldsymbol{j}}-\boldsymbol{H} \boldsymbol{w}_{\boldsymbol{i}}\right)\right\} \\
>\frac{\sqrt{\rho_{B}}}{2}\left\|\boldsymbol{H} \boldsymbol{w}_{\boldsymbol{i}}-\boldsymbol{H} \boldsymbol{w}_{\boldsymbol{j}}\right\|^{2}
\end{array}\right) \\
& =Q(\sqrt{\mu}),
\end{aligned}
$$

where $\Re\{\cdot\}$ represents the real part of its argument, $Q(x)=$ $\frac{1}{\sqrt{2 \pi}} \int_{x}^{\infty} e^{-\frac{t^{2}}{2}} d t$, and

$$
\mu=\frac{\rho_{B}}{2 N_{0}}\left\|\boldsymbol{H} \boldsymbol{w}_{\boldsymbol{i}}-\boldsymbol{H} \boldsymbol{w}_{\boldsymbol{j}}\right\|^{2} .
$$

Since $\boldsymbol{H}$ is Gaussian distributed, $\mu$ in (6) is the summation of $2 N_{r}$ independent random variables expressed as

$$
\mu=\sum_{i=1}^{2 N_{r}} \alpha_{i}^{2}
$$


where $\alpha_{i} \sim \mathcal{N}\left(0, \sigma_{\alpha}^{2}\right)$ with $\sigma_{\alpha}^{2}=\frac{\rho_{B}}{2 N_{0}} \triangleq \sigma_{\alpha_{1}}^{2}$ for $\boldsymbol{w}_{\boldsymbol{j}} \in \mathcal{W}^{\prime}$ and $\sigma_{\alpha}^{2}=\frac{\rho_{B}}{N_{0}} \triangleq \sigma_{\alpha_{2}}^{2}$ for $\boldsymbol{w}_{j} \in \mathcal{W}^{\prime \prime}$. Note that the above results are still valid for the special cases $(q=m$ or $p=n)$ $[9$, p. 49].

Since $\mu$ is chi-squared distributed with $2 N_{r}$ degrees of freedom, the probability density function (PDF) of $\mu$, denoted by $p_{\mu}(x)$, can be obtained [9, p. 41]. Thus, by averaging over all channel realizations the PEP is given by

$$
\begin{aligned}
P\left(\boldsymbol{w}_{\boldsymbol{i}} \rightarrow \boldsymbol{w}_{\boldsymbol{j}}\right) & =\mathbb{E}_{\mu}\left[P\left(\boldsymbol{w}_{\boldsymbol{i}} \rightarrow \boldsymbol{w}_{\boldsymbol{j}} \mid \boldsymbol{H}\right)\right] \\
& =\int_{v=0}^{\infty} Q(\sqrt{v}) p_{\mu}(v) d v .
\end{aligned}
$$

The closed form expression of (8) can be obtained as [10, Eq. (64)]

$$
P\left(\boldsymbol{w}_{\boldsymbol{i}} \rightarrow \boldsymbol{w}_{\boldsymbol{j}}\right)=\gamma_{\alpha}^{N_{r}} \sum_{t=0}^{N_{r}-1}\left(\begin{array}{c}
N_{r}-1+t \\
t
\end{array}\right)\left[1-\gamma_{\alpha}\right]^{t},
$$

where $\gamma_{\alpha}=\frac{1}{2}\left(1-\sqrt{\frac{\sigma_{\alpha}^{2}}{1+\sigma_{\alpha}^{2}}}\right)$. Replacing the two PEP in (4) with (9), and using the cardinality of $\mathcal{W}^{\prime}$ and $\mathcal{W}^{\prime \prime}$, the SER of BiSSK is given by

$$
\begin{aligned}
P_{\text {Sym }} & \leq 2\left(N_{t}-1\right) \gamma_{\alpha_{1}}^{N_{r}} \sum_{t=0}^{N_{r}-1}\left(\begin{array}{c}
N_{r}-1+t \\
t
\end{array}\right)\left[1-\gamma_{\alpha_{1}}\right]^{t} \\
& +\left(N_{t}-1\right)^{2} \gamma_{\alpha_{2}}^{N_{r}} \sum_{t=0}^{N_{r}-1}\left(\begin{array}{c}
N_{r}-1+t \\
t
\end{array}\right)\left[1-\gamma_{\alpha_{2}}\right]^{t},
\end{aligned}
$$

where

$\gamma_{\alpha_{1}}=\frac{1}{2}\left(1-\sqrt{\frac{\sigma_{\alpha_{1}}^{2}}{1+\sigma_{\alpha_{1}}^{2}}}\right)$ and $\gamma_{\alpha_{2}}=\frac{1}{2}\left(1-\sqrt{\frac{\sigma_{\alpha_{2}}^{2}}{1+\sigma_{\alpha_{2}}^{2}}}\right)$.

The analytical upper bound on the SER of BiSSK modulation in (10) will be plotted along with empirical results in Sec. V.

Diversity: The diversity of BiSSK modulation can be derived by using a looser upper bound. Using the Chernoff bound $Q(x) \leq \frac{1}{2} \exp \left(-\frac{x^{2}}{2}\right)$ [9, p. 54], we have [1]

$$
\begin{aligned}
P\left(\boldsymbol{w}_{\boldsymbol{i}} \rightarrow \boldsymbol{w}_{\boldsymbol{j}}\right) & \leq \int_{0}^{\infty} \frac{\exp \left(-\frac{v}{2}\right) v^{N_{r}-1} \exp \left(-\frac{v}{2 \sigma_{\alpha}^{2}}\right)}{2^{N_{r}+1} \sigma_{\alpha}^{N_{r}} \Gamma\left(N_{r}\right)} d v \\
& =\frac{1}{2}\left(\sigma_{\alpha}^{2}+1\right)^{-N_{r}} \\
& \leq \frac{1}{2}\left(\sigma_{\alpha}^{2}\right)^{-N_{r}},
\end{aligned}
$$

where $\sigma_{\alpha}^{2}=\frac{\rho_{B}}{2 N_{0}}$ for $\boldsymbol{w}_{\boldsymbol{j}} \in \mathcal{W}^{\prime}$ and $\sigma_{\alpha}^{2}=\frac{\rho_{B}}{N_{0}}$ for $\boldsymbol{w}_{\boldsymbol{j}} \in$ $\mathcal{W}^{\prime \prime}$. As a result, we have $P_{\text {sym }} \leq C\left(\frac{E_{s}}{N_{0}}\right)^{-N_{r}}$, where $C$ is a constant. Thus, the diversity order of BiSSK is $N_{r}$.

\section{Simulations AND Discussions}

This section presents the simulation results for uncoded SSK-based transmission systems. Optimal ML detection is adopted at the receiver.

Comparison with SSK: Note that $\rho$ in (1) can be expressed as $\rho=E_{b} N_{b} / \mathbb{E}\left[\boldsymbol{x}^{H} \boldsymbol{x}\right]$, where $E_{b}$ and $N_{b}$ represent the bit energy and the number of bits per symbol, respectively. Given a fixed number of transmitting antennas $N_{t}$, BiSSK achieves twice the transmission rate ( $N_{b}$ bits per symbol) as SSK while also requiring twice the symbol power $\left(\mathbb{E}\left[\boldsymbol{x}^{H} \boldsymbol{x}\right]\right)$. Therefore,

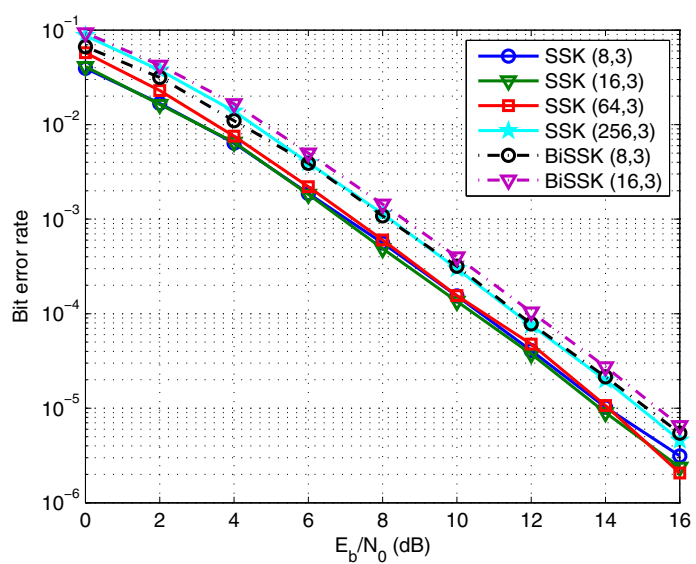

Fig. 1. The BER performance of BiSSK versus SSK with $\left(N_{t}, N_{r}\right)$.

in order to make a fair comparison of BiSSK and SSK, we show the bit error rate (BER) vs. $E_{b} / N_{0}$ (rather than $E_{s} / N_{0}$ ) performance.

The BERs of SSK-based modulation systems with $\left(N_{t}, N_{r}\right)$ transmitting/receiving antennas are shown in Fig. 1. At a fixed number of transmitting antennas, e.g., $N_{t}=8$, BiSSK doubles the transmission rate of SSK at the expense of $1 \mathrm{~dB}$ performance loss when BER $=10^{-5}$. At a fixed target transmission rate, e.g., 8 bits/s/Hz for both $\operatorname{BiSSK}(16,3)$ and SSK $(256,3)$, BiSSK shows negligible performance loss. In achieving the same transmission rate, BiSSK requires only a square-root number of transmitting antennas as compared to SSK, which makes BiSSK highly favorable due to the significantly reduced hardware cost (the number of transmitting antennas) especially for high-rate transmission.

Here, we conduct error rate comparison between SSK and BiSSK by analyzing the worst-case error symbol. Given fixed $N_{t}, N_{r}$, and a specific realization of $\boldsymbol{H}$, there are two distinct columns of $\boldsymbol{H}$ with the smallest Euclidean distance in between, denoted by $\boldsymbol{h}_{\boldsymbol{k}}$ and $\boldsymbol{h}_{\boldsymbol{s}}(k \neq s)$, where $\boldsymbol{h}_{\boldsymbol{l}}$ represents the $l$-th column of $\boldsymbol{H}$. The worst-case error symbol is likely to occur at the erroneous detection between two channel columns with smallest Euclidean distance, i.e., $\boldsymbol{h}_{\boldsymbol{k}}$ and $\boldsymbol{h}_{\boldsymbol{s}}$. Denoting the transmitted symbol and worst-case error symbol as $\boldsymbol{x}_{\boldsymbol{k}}$ and $\boldsymbol{x}_{\boldsymbol{s}}$, the distance between $\boldsymbol{x}_{\boldsymbol{k}}$ and $\boldsymbol{x}_{\boldsymbol{s}}$ is given by

$$
\begin{aligned}
d_{S S K} & =\left\|\sqrt{\rho} \boldsymbol{H}\left(\boldsymbol{x}_{\boldsymbol{k}}-\boldsymbol{x}_{\boldsymbol{s}}\right)\right\| \\
& =\sqrt{E_{b} \log _{2} N_{t}}\left\|\boldsymbol{h}_{\boldsymbol{k}}-\boldsymbol{h}_{\boldsymbol{s}}\right\|,
\end{aligned}
$$

where minimum distance can be understood as the ability to resist noise. For BiSSK, the worst-case error symbol corresponds to the error occurring between symbols $\boldsymbol{w}_{\boldsymbol{i}}$ and $\boldsymbol{w}_{\boldsymbol{j}}$ whose indices have the following relation

$$
\begin{cases}\text { either } & p=m, q \neq n, \\ \text { or } & p \neq m, q=n .\end{cases}
$$

That is, the worst-case error symbol is in the set $\mathcal{W}^{\prime}$. As a result, the minimum distance for BiSSK is given by

$$
d_{B i S S K}=\sqrt{\frac{E_{s}}{2}}\left\|\boldsymbol{h}_{\boldsymbol{k}}-\boldsymbol{h}_{\boldsymbol{s}}\right\|=\sqrt{E_{b} \log _{2} N_{t}}\left\|\boldsymbol{h}_{\boldsymbol{k}}-\boldsymbol{h}_{\boldsymbol{s}}\right\| .
$$




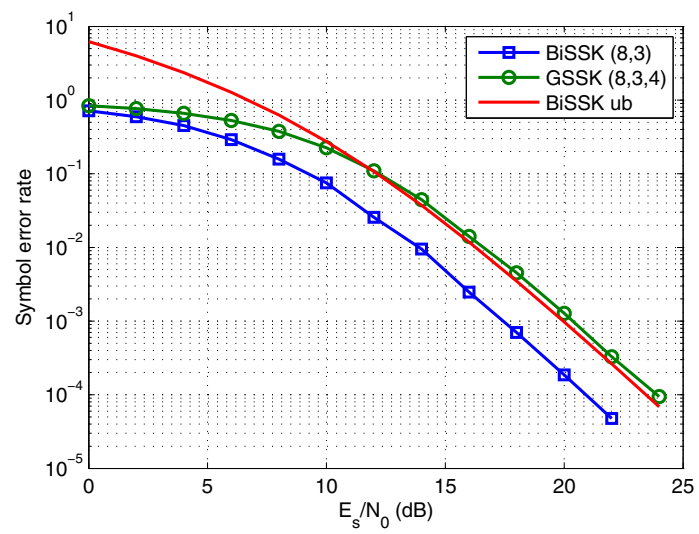

Fig. 2. The SER performance of BiSSK $\left(N_{t}, N_{r}\right)$ versus GSSK $\left(N_{t}, N_{r}, n_{t}\right)$, where the analytical upper bound of BiSSK is shown as well. This simulation targets at $6 \mathrm{bits} / \mathrm{s} / \mathrm{Hz}$ transmission.

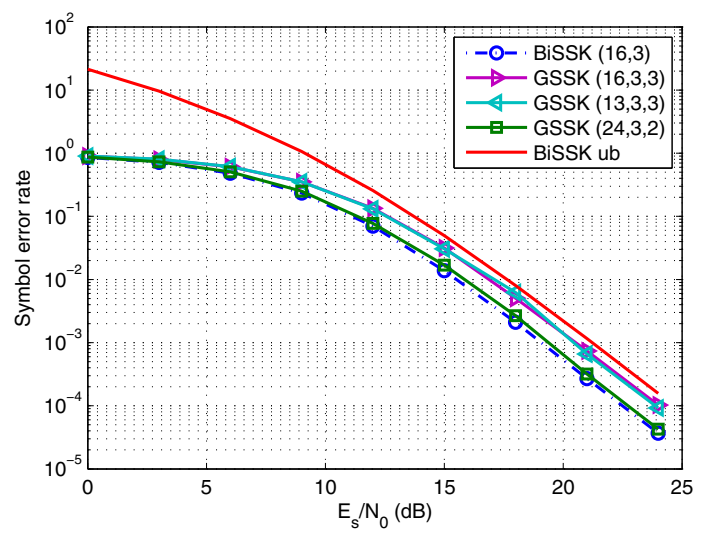

Fig. 3. The SER performance of BiSSK $\left(N_{t}, N_{r}\right)$ versus GSSK $\left(N_{t}, N_{r}, n_{t}\right)$, where the analytical upper bound of BiSSK is shown as well. This simulation targets at $8 \mathrm{bits} / \mathrm{s} / \mathrm{Hz}$ transmission.

As can be seen, $d_{B i S S K}=d_{S S K}$ in the worst case. Since this result applies to every channel realization, it suggests that the PEP of SSK and BiSSK are comparable.

Comparison with GSSK: Both GSSK [7] and BiSSK aim to enhance the transmission rate of SSK under limited number of transmitting antennas. Let GSSK be equipped with $\left(N_{t}, N_{r}, n_{t}\right)$ transmitting/receiving/activated antennas and BiSSK be equipped with $\left(N_{t}, N_{r}\right)$ transmitting/receiveing antennas. The simulation results with fixed $6 \mathrm{bits} / \mathrm{s} / \mathrm{Hz}$ transmission and 8 bits/s/Hz transmission are shown in Fig. 2 and Fig. 3, respectively. In Fig. 2, we observe that an approximate $3 \mathrm{~dB}$ gain is achieved by BiSSK at SER $=10^{-4}$. In Fig. 3, GSSK with $n_{t}=3$ and $n_{t}=2$ are shown for comparison. GSSK with $n_{t}=3$ requires the same or smaller number of transmitting antennas to achieve the same transmission rate as BiSSK. On the other hand, BiSSK demonstrates an approximate $1.5 \mathrm{~dB}$ gain over GSSK with $n_{t}=3$ at $\mathrm{SER}=10^{-4}$. GSSK with $n_{t}=2$ shows comparable performance as BiSSK yet at the cost of a larger $N_{t}(24)$.

The worst-case analysis is used again to compare the performances of GSSK and BiSSK. The same model as (12) is adopted except that BER is replaced by $\operatorname{SER}\left(E_{b} N_{b}\right.$ is replaced by $E_{s}$ ). The minimum distance between GSSK constellation points is

$$
d_{G S S K}=\sqrt{\frac{E_{s}}{n_{t}}}\left\|\boldsymbol{h}_{\boldsymbol{k}}-\boldsymbol{h}_{\boldsymbol{s}}\right\| .
$$

Comparing (14) with the second term in (13), it is seen that the minimum distance of GSSK depends on $n_{t}$. This explains why the performance of BiSSK exceeds GSSK in our simulations (since if $n_{t}>2, d_{G S S K}<d_{B i S S K}$ ). Furthermore, since the required number of RF chains at the transmitter is two for BiSSK and $n_{t}$ for GSSK, BiSSK has an inexpensive RFelement requirement compared to GSSK while achieving the same transmission rate.

\section{CONCLUSION}

In this letter, an SSK-based modulation scheme is proposed. The advantages of the proposed BiSSK are listed as follows. 1) The BiSSK utilizes the antenna indices to convey information. Therefore, the proposed BiSSK preserves the advantages of low receiver complexity of SSK. 2) Given the same number of transmitting antennas $N_{t}$, the proposed BiSSK achieves twice the transmission rates of SSK (at negligible performance loss). Conversely, given the same target transmission rate, BiSSK requires a square-root number of transmitting antennas as compared with SSK. This significantly reduces the hardware cost when high transmission rates using SSK-based modulation are desired. 3) BiSSK outperforms GSSK in achieving the same transmission rates, as demonstrated by the analysis, simulation, and hardware discussion in Sec. V.

\section{REFERENCES}

[1] J. Jeganathan, A. Ghrayeb, L. Szczecinski, and A. Ceron, "Space shift keying modulation for MIMO channels," IEEE Trans. Wireless Commun., vol. 8, no. 7, pp. 3692-3703, July 2009.

[2] M. D. Renzo and H. Haas, "Transmit-diversity for spatial modulation (SM): towards the design of high-rate spatially-modulated space-time block codes," in Proc. 2011 IEEE Int. Conf. Commun., pp. 1-6.

[3] S. Sugiura, S. Chen, and L. Hanzo, "Generalized space-time shift keying designed for flexible diversity-, multiplexing- and complexity-tradeoffs," IEEE Trans. Wireless Commun., vol. 10, no. 4, pp. 1144-1153, Apr. 2011.

[4] M. D. Renzo and H. Haas, "Improving the performance of space shift keying (SSK) modulation via opportunistic power allocation," IEEE Commun. Lett., vol. 14, no. 6, pp. 500-502, June 2010.

[5] N. Serafimovski, M. D. Renzo, S. Sinanovic, R. Y. Mesleh, and H. Haas, "Fractional bit encoded spatial modulation (FBE-SM)," IEEE Commun. Lett., vol. 14, no. 5, pp. 429-431, May 2010.

[6] R. Y. Mesleh, H. Haas, S. Sinanovic, C. W. Ahn, and S. Yun, "Spatial modulation," IEEE Trans. Veh. Technol., vol. 57, no. 4, pp. 2228-2241, July 2008.

[7] J. Jeganathan, A. Ghrayeb, and L. Szczecinski, "Generalized space shift keying modulation for MIMO channels," in Proc. 2008 IEEE Int. Symp. Personal, Indoor and Mobile Radio Commun., pp. 1-5.

[8] R. Y. Chang, S.-J. Lin, and W.-H. Chung, "New space shift keying modulation with Hamming code-aided constellation design," IEEE Wireless Commun. Lett., vol. 1, no. 1, pp. 2-5, Feb. 2012.

[9] J. G. Proakis, Digital Communications, 4th edition. McGraw-Hill, 2001.

[10] M.-S. Alouini and A. J. Goldsmith, "A unified approach for calculating error rates of linearly modulated signals over generalized fading channels," IEEE Trans. Commun., vol. 47, no. 9, pp. 1324-1334, Sep. 1999. 